\title{
Cardiac Arrhythmia Classification by Wavelet Transform
}

\author{
Hadji Salah $^{1}$ \\ TIC department \\ LR SITI ENIT \\ BP 37 Belvédère 1002 Tunis, Tunisia
}

\author{
Ellouze Noureddine ${ }^{2}$ \\ TIC department \\ LR SITI ENIT \\ BP 37 Belvedere 1002 Tunis, Tunisia
}

\begin{abstract}
Cardiovascular diseases are the major public health parameter; they are the leading causes of mortality in the world. In fact many studies have been implemented to reduce the risk, including promoting education, prevention, and monitoring of patients at risk. In this paper we propose to develop classification system heartbeats. This system is based mainly on Wavelet Transform to extract features and Kohonen selforganization map the arrhythmias are considered in this study : N,(Normal), V(PrematureVentricular),A(AtrialPremature),S(Ext rasystolesupraventriculaire),F(FusionN+S),R(RightBundle Branch) .
\end{abstract}

Keywords-ECG; QT database; Wavelet Transform; Classification; Kohonen self-organization map

\section{INTRODUCTION}

The ECG signal is a representation of the electrical heart activity; it is used to analyze the status of the heart. Due to the morphological variability of the different waves of ECG and the presence of noise that interfere with the ECG signal it is so difficult to extract necessary information's from the signal. These difficulties require tools of signal processing and automatic classification of cardiac Arrhythmia. Therefore, many researches were proposed for the automatic classification of the signal heart many methods have been implemented, such as the statistic approach, fuzzy logic and neural networks. In our paper, we have opted for neural classifiers because of their efficiencies were solving problems of classification in the case of large databases dimensions. As classifier, we chose to use the Kohonen topological maps [7,8,9] receives as input 12 parameters characterizing a temporal and morphological ECG beat are mainly: (Length $(\mathrm{QRS})$, amp $(\mathrm{Q}, \mathrm{R}, \mathrm{S})$ intervals $(\mathrm{QT}$, $\mathrm{PR}, \mathrm{RR})$ ). In this article, we present in Section II the steps and algorithms used for extracting the characteristic parameters of a heart beat. In Section III, we will describe the methodology adopted for the development of our system classification. In Section IV, we present the main results. A conclusion and outlook for this work are given in Section V.

\section{WAVELET TRANSFORM}

Wavelet Transform is a time-scale representation of signals that has been used in a wide range of applications, including signal analysis and compression. Recently, wavelets have been applied to several problems in Electro cardiology, including data compression, analysis of ventricular late potentials and time localization of ECG characteristics. The Wavelet Transformation (WT) is a linear operation that decomposes a signal into a number of scales related to frequency components and represents each scale with a fixed time and frequency resolution [12]. WT analysis uses a short time interval for evaluating high frequencies and a long time interval for low frequencies. Wavelet transform of a signal $f(t)$ is defined as the sum of over all time of the signal multiplied by scaled versions of the wavelet function $\psi$, and is given by :

$$
\begin{aligned}
& w_{a, b}=\int_{-\infty}^{+\infty} f(t) \psi_{a, b}(t) \delta t \\
& \psi_{a, b}(t)=\frac{1}{\sqrt{a}} \psi *\left(\frac{t-b}{a}\right)
\end{aligned}
$$

Where $*$ denotes complex conjugation of the mother wavelet $\psi_{a, b}(t)$ and $\psi\left(\frac{t-b}{a}\right)$ is the shifted and scaled version, the constant $\frac{1}{\sqrt{a}}$ is an energy normalization factor. When the scale parameter is the set of integral powers of 2 , i.e. $\mathrm{a}=2^{j} \quad(\mathrm{j} \in \mathrm{z}, \mathrm{z}$ is an Integer set). The wavelet transform is called dyadic wavelet transform. The wavelet transform at scale $2^{j}$ is given by:

$$
W f\left(2^{j}, \tau\right)=\frac{1}{\sqrt{2}} \int_{-\infty}^{+\infty} f(t)\left(\frac{t-\tau}{2^{j}}\right) \delta \tau
$$

\section{A. Stationary wavelet transform}

The stationary wavelet transform (SWT: Stationary Wavelet Transform) is an intermediate representation of views between the redundant CWT high redundancy and nonredundant DWT. It retains an almost dyadic sampling continuous and uniform time [12] [59], the coefficients of its decomposition have the appearance of the filtered signals in contrast to the DWT [12]. The SWT is defined by calculating the coefficients: $c d_{x}(j, k)_{(j, k) \in z^{2}}$

$$
\begin{aligned}
& c d \cdot_{x}(j, k)=\int_{R} x(t) \psi^{*}{ }_{j, k}(t) d t \\
& \text { Where } \psi(t)=2^{\frac{-j}{2}} \psi_{0,0}\left(2^{-j}(t-k)\right)
\end{aligned}
$$

the relationship between the wavelet bases used in the DWT and SWT is implemented using the following wording:

$$
\psi_{j, k} \psi_{j, 0}\left(t-2^{j} k\right), \psi_{j, k}^{\prime}(t)=\psi_{j, 0}(t-k) \quad \text { (5) with }
$$




$$
\psi_{j, 0}(t)=2^{\frac{-j}{2}} \psi\left(\frac{t}{2^{j}}\right)
$$

and thus the SWT coincides with the CWT at the uniform grid and the grid dyadic DWT where $c d_{x}(j, k)$ have the same frequency resolution as $d x(j, k)$, but sharing information on the time axis

$$
\begin{aligned}
c d_{x}(j, k) & =c w t_{x}\left(a=2^{j}, t=k\right) \\
c d_{x}\left(j, 2^{j} k\right) & =d_{x}(j, k)
\end{aligned}
$$

The SWT is. Translation invariant in time, what is missing in the DWT and these results from the fact that it has a time sampling of the same signal. This explains the $\mathrm{cd}_{\mathrm{j}}$ have the properties of the filtered signal

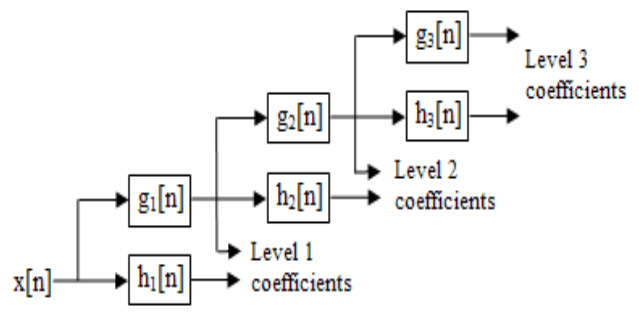

Fig. 1. 3 level decomposition

\section{B. QT database:}

The QT is a basic standard database available on the website PhysioNet, which is an international reference in the field of ECG signal processing [11]. The database QT PhysioNet was established by researchers to be used as a reference for validation and comparison of algorithms for segmentation of the ECG. It is known to have a low signal-tonoise ratio with different pathologies leading to accurate detection of waves through this entire base. Therefore, at present, the basic QT is the only database that is annotated appropriately to test our analysis method of long-term recordings. It contains 105 two-channel Holter-recordings primarily (ITNs and V5) of 15 minutes sampled at $250 \mathrm{~Hz}$. These ECG signals were extracted from different databases already existing such as the database arrhythmias MIT-BIH, the database ST-T of the European Society of Cardiology, and several other databases assembled by Boston's Beth medical center

\section{SOM SELF-ORGANIZATION MAP}

SOM (Self-organizing Map) is an artificial neural network (ANNA) architecture based on unsupervised, competitive learning [14]. It provides a topology preserving, smooth mapping from a high-dimensional input space to the map units usually arranged as a two-dimensional lattice of neurons (nodes). Thus, the SOM can serve as a tool for cluster analysis of complex, high-dimensional data. A parametric reference vector $\mathrm{m}$ is associated with every node. A data vector $\mathrm{x}$ is compared to all reference vectors in any metric and the best matching node is defined, e.g., by the smallest Euclidean distance between the data vector and any of the reference vectors. During learning, those nodes that are topographically close in the array up to a certain distance will activate each other to learn from the same input:

$$
m_{i}(t+1)=m_{i}(t)+h_{c i}(t) \cdot\left[x(t)-m_{i}(y)\right]
$$

where $t$ is an integer representing time, and $h_{c i}$ is the so-called neighborhood kernel describing the neighborhood that is updated around the best-matching node. Several suitable kernels can be used, e.g. a so-called bubble kernel or a Gaussian kernel, relating to different ways of determining the activating cells. The kernel also includes the learning rate parameter) ( $\mathrm{t} \alpha$. With time, the size of the neighborhood and the learning rate are diminished. The described learning process leads to a smoothing effect on the weight vectors in the neighborhood and by continued learning to global ordering of the nodes [14]

The SOM consists of a two-dimensional lattice that contains a number of neurons. These neurons are usually arranged in a rectangular or hexagonal way. The position of the units in the grid, especially the distances between them and the neighborhood relations, are very important for the learning algorithm. A prototype vector (also "model" or "codebook" vector) is associated with each neuron, which is a vector of the same dimension as the input data set. This prototype vector approximates a subset of the sample vectors. The dimension of the sample is called input dimension, and is usually larger than 2 , the dimension of the lattice, which is called output dimension. For training and visualization purposes, the sample vectors are assigned to the most similar prototype vector, or best-matching unit (BMU), formally

$$
C(x)=\arg \min \left\{\left|x-m_{i}(t)\right|\right\}
$$

The learning process itself gradually adapts the model vectors to match the samples and to reflect their internal properties as faithfully as possible, which means that input vectors which are relatively close in input space should be mapped to units that are relatively close on the lattice.

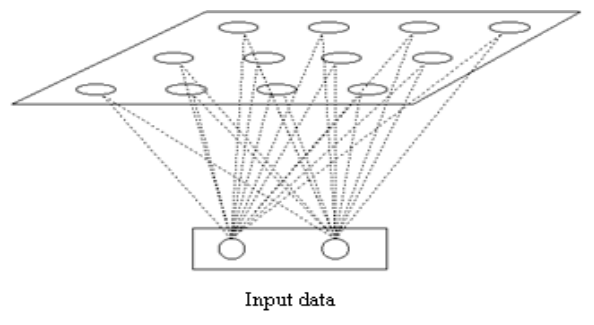

Fig. 2. Kohonen Network map

\section{A. Parameters extraction}

Each ECG beat, consisting of 376 samples centered on the position of the R wave undergoes a SWT on four successive scales using wavelet (db4) as mother wavelet. Subsequently, we calculate the energy of the Stationary wavelet transform each level. Thus it reduces the size of the input vector of 376 elements only 8 for the 4 th level gave the best recognition rate. The classification scheme is shown in Figure 3. 


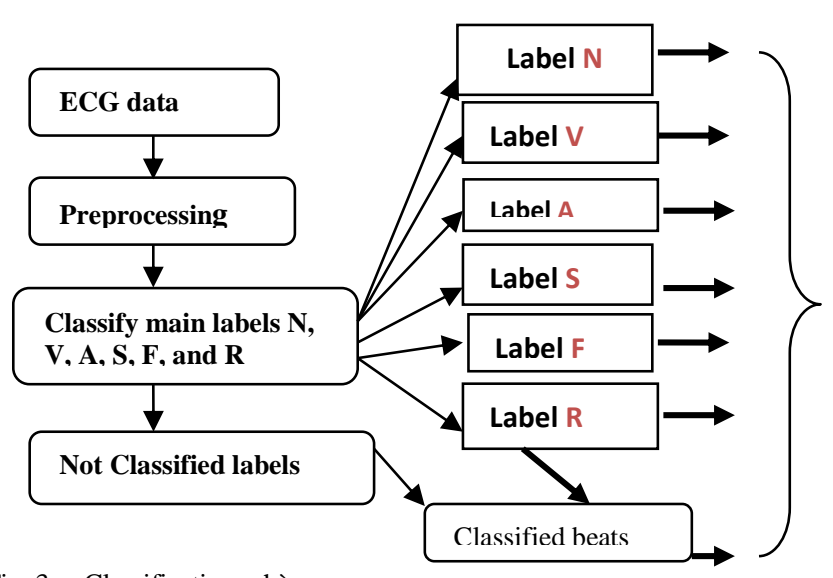

Fig. 3. Classification schème

\section{B. Performance and Measures}

The performance of the classification system was measured based on tow standard statistical measures for each class: Sensitivity (Se) and Predectivity ( $\mathrm{Sp}$ ) which are calculated from multi-class classification table [1].

\section{RESULTS}

TABLE I. CONFUSION- MATRIX

\begin{tabular}{|l|l|l|l|c|c|c|}
\hline & $\mathbf{N}$ & $\mathbf{V}$ & $\mathbf{A}$ & $\mathbf{S}$ & $\mathbf{F}$ & $\mathbf{R}$ \\
\hline $\mathbf{N}$ & 26792 & 23 & 455 & 458 & 125 & 360 \\
\hline $\mathbf{V}$ & 0 & 562 & 0 & 0 & 35 & 6 \\
\hline $\mathbf{A}$ & 0 & 0 & 365 & 0 & 4 & 3 \\
\hline $\mathbf{S}$ & 1 & 5 & 1 & 235 & 3 & 1 \\
\hline $\mathbf{F}$ & 2 & 0 & 0 & 0 & 58 & 0 \\
\hline $\mathbf{R}$ & 4 & 3 & 0 & 0 & 0 & 123 \\
\hline
\end{tabular}

\begin{tabular}{|l|l|l|}
\hline & Se (\%) & Sp (\%) \\
\hline $\mathbf{N}$ & 94,7 & 99,98 \\
\hline $\mathbf{V}$ & 93,7 & 94,7 \\
\hline $\mathbf{A}$ & 97,4 & 98,3 \\
\hline $\mathbf{S}$ & 95,4 & 98,3 \\
\hline $\mathbf{F}$ & 96,6 & 96.6 \\
\hline $\mathbf{R}$ & 94,4 & 94,1 \\
\hline
\end{tabular}

Fig. 4. displayed results with SOM

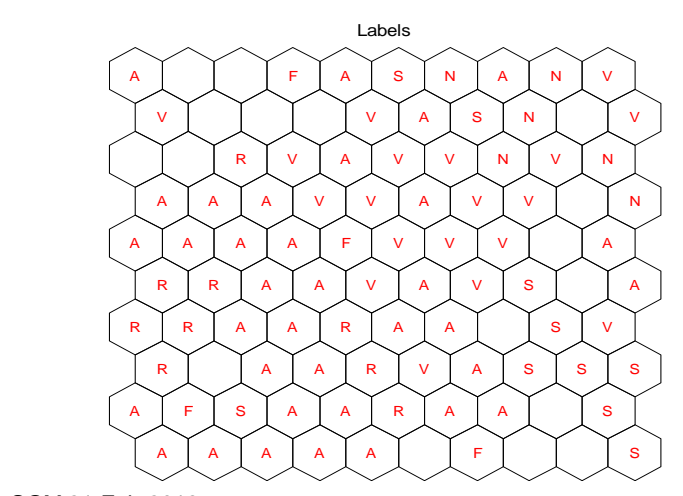

SOM 21-Feb-2013

Fig. 5. cardiac Arrhythmia recognition and displayed by Kohonen map

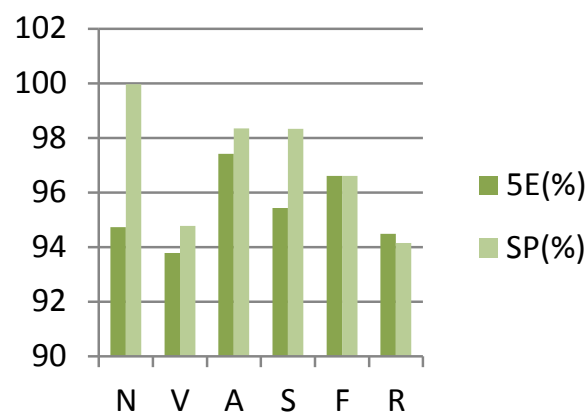

Fig. 6. Histogram variation of recognition rates by wavelet Transform

\section{CONCLUSION}

In this work we have developed a new method based on wavelet transform decomposition and self organizing map algorithm to classify cardiac arrhythmia labels such as N, A, V, $\mathrm{R}, \mathrm{F}, \mathrm{S}$, obtained results are very interesting .

The specificity and the Predectivity were higher than $94 \%$ in all cases $(\mathrm{N}, \mathrm{A}, \mathrm{S}, \mathrm{R}, \mathrm{V}$ and $\mathrm{F})$. This result means that the proposed method is suitable enough for practical clinical use.

[1] Physiobank Archive Index, QT Database. http://www.physionet.org/physiobank/database

[2] S.Z.Mahmoodabadi,A.Ahmadian and M D. Abolhasan,"ECG Feature Extraction using Daubechies Wavelets", Proceedings of the fifth IASTED International conference on Visualization, Imaging and Image Processing, pp. 343-348, 2005.

[3] Palreddy, S., Hu, Y.H. and Tompkins, W.J. "A patient adaptable ECG beat classifier using a mixture of experts approach", IEEE Trans. on BME, 44(9), 1997, 891-900.

[4] Acr, N. "Classification of ECG beats by using a fast least square support vector machines with a dynamic programming feature selection algorithm", Neural Computing and Applications, 14(4), 2005, 299-309.

[5] P. de Chazal, M. O'Dwyer, and R. Reilly, "Automatic classification of heartbeats using ECG morphology and heartbeat interval features," IEEE Trans. Biomed. Eng., vol. 51, no. 7, pp. 1196-1206, Jul. 2004.

[6] Übeyli, E.D., ECG beats classification using multiclass support vector machines with error correcting output codes Digital Signal Processing, Volume 17, Issue 3, May 2007, 675-68

[7] Osowski, S. and Linh, T.R. "ECG Beat Recognation Using Fuzzy Hybrid Neural Network", IEEE Trans. on BME, 48(11), 2001.

[8] Nadal, J. and Bossan, M. "Classification of cardiac arrhythmia based on principal components analysis and feedforward neural Networks", Comput Cardiol, 1993, 341-344.

[9] Hosseini, H.G., Reynolds, K.J., and Powers, D., "A Multi-stage Neural Network Classifier for ECG Events", Proceedings of the 23rd International Conference of the IEEE Engineering in Medicine and Biology Society, 2001, October 25-28.

[10] Minami, K., Nakajima, H. and Toyoshima, T. "Arrhythmia diagnosis with discrimination of rhythm origin and measurement of heart-rate variation", Comp Cardiol,1997,243-246.

[11] Kohonen, T., (2001). Self-Organizing Maps, 3rd ed., Heidelverg: Springer-Verlag Berlin.

[12] Salah Hadji, "Caractérisation du complexe QRS du signal ECG et Identification des arythmies cardiaques", Thèse de doctorat de l'université de Tunis Elmanar 3,2012

[13] Kohonen, T.: Self-organizing Maps, Springer-Verlag,1995.

[14] [1Kohonen,T.,Hynninen,J.,Kangas,J.Laaksonen,J.:SOM_PAK - The Self-organizing Map Program Package, 1995. 